\title{
REDUCTION OF UNCERTAINTIES IN LASER STRIPE MEASUREMENT OF SOLID PARTICLES CONCENTRATION
}

\author{
Jan KrupiČKA*, Tomáš PICEK, ŠTĚPÁn ZRostlíK \\ Czech Technical University in Prague, Faculty of Civil Engineering, Thákurova 7, Prague, Czech Republic \\ * corresponding author: jan.krupicka@fsv.cvut.cz
}

\begin{abstract}
Laser stripe measurement (LSM) is a relatively novel method for measuring a local concentration of coarse particles in a mixture with fluid. It is based on an analysis of camera records of the laser sheet penetration in to the mixture. We report on our measurements of the concentration of suspended particles in a fluidization cell and focus on the parameters affecting the evaluation procedure for the measured data. A high sensitivity of the measured concentration to the correction for the position of the wall and to the threshold brightness for data filtering is demonstrated. The uncertainty in the wall position is reduced by applying a rectification procedure based on an identification of the position of a laser stripe drawn at the wall of the fluidization cell. The main motivation for the presented study was to find guidelines for the determination of the threshold brightness, absence of which can be considered a serious weakness of the LSM when particles of non-ideal optical properties are tested. Histograms of the brightness of laser stripes drawn on a surface of suspended particles are analysed with the aim to find a connection between the histograms and the threshold brightness. The threshold brightness is shown to be proportional to a position of the second of the two peaks identified in a histogram. Based on the results of the analysis, a method is proposed for the determination of the threshold brightness.
\end{abstract}

KEYWORDS: laser stripe measurement; coarse particle concentration; solid fraction measurement; fluidization cell; brightness threshold.

\section{INTRODUCTION AND MOTIVATION}

An inherent part of our research project in the field of the open channel flow of settling slurries is an experimental work focused on an investigation of the inner structure of the flow, which can be characterised by a presence of layers with different particle concentrations, velocities and mechanisms of particle and fluid interactions. The aim of the experimental work is to provide the information necessary for understanding relations between the local concentration, velocity and fluid/solids stresses and for developing and testing appropriate mathematical models [1-4]. Besides other things, we need to measure vertical profiles of the local volumetric concentration of solid particles carried by water in an experimental flume. The following specific characteristics of the flow make the measurement of the concentration difficult: flow (and particle) velocities differ considerably across the flow; the flow is opaque due to a high concentration in lower layers; and - what is probably the most limiting feature the use of relatively large particles leads to the fact that the desired spatial resolution of the concentration measurement is comparable with the size of the particles. This disqualifies a direct use of traditional methods of measurement, such as isokinetic sampling and measurement of local electrical properties of the mixture. In [5], Spinewine et al. proposed an optical method, which seems to be successful under the conditions described above. However, there are certain parameters of the method, which are difficult to evaluate and which affect results considerably as will be discussed below. This causes that this promising method lacks of robustness. The motivation of the presented study was to improve the method performance by reducing its sensitivity to the measurement errors and to a priori experience.

\section{LASER STRIPE MEASUREMENT METHOD}

The Laser Stripe Measurement (LSM) method was originally proposed in [5] where details can be found. In the following section, we provide only a brief overview of the basic principles necessary for the discussion of the method performance.

\subsection{Principles OF THE MEthod}

The method is based on the measurement of the depth of a penetration of a laser sheet to the flow. The laser sheet penetrates the flow via a transparent side wall of a conduit and the plane of the sheet is oriented perpendicular to both the wall and the flow direction. In the case of the open flume with a longitudinal axis $x$, the laser sheet plane is defined by a vertical axis $z$ and transverse axis $y$. The higher is the concentration of particles at a given vertical position of the flume, the higher is the probability that the laser hits some particle near the wall - in other words, the smaller is the mean optical free path $\mu$ of the laser within the flume. Distance from the wall, $y$, of a particle 
illuminated by the laser sheet can be evaluated from an image shot in a direction deviated from the sheet plane by an angle $\alpha$. To obtain relevant statistics on the mean free path of the laser sheet, a camera is used to acquire a large number of images of bright stripes drawn by the laser on the surface of particles travelling through the sheet plane. The procedure of measuring and data evaluation can be described in following steps:

(1.) Firstly, calibration image is taken of a target placed within the flume. The known geometry of the target is then used to calibrate parameters of the holomorphic transformation between the column and row index of the pixel in the image and physical coordinates $[y, z]$ of the corresponding point within the flume cross-section illuminated by the laser.

(2.) Cartesian mesh of positions $[y, z]$ is defined within the flume cross-section with an intersection with the wall given by the line $y=0$. Then, a binning map $M(y, z)$ is constructed, which stores information on a count of particle hits observed at each position $[y, z]$ during experiment: for each image recorded by the camera, a holomorphic transformation is employed to project the mesh $[y, z]$ to the image, the point of maximal brightness $B$ is identified at each $z$-position, $y$-position of this point is calculated and value of the $M(y, z)$ is increased by one. Due to occlusion effects, not all particles illuminated by the laser are observed by the camera and hence not all identified points of maximal brightness correspond to a laser hit (some of them correspond to reflections only). Therefore, only points of brightness higher than a certain threshold value $B_{\text {th }}$ are considered as an image of laser hits and counted to the binning map $M$; points of $B<B_{\text {th }}$ are considered a "false" hits and discarded from the analysis.

(3.) Mean y-position of the observed particle hit by laser, $\mu_{\mathrm{obs}}$, is calculated for each z-position from the binning map. The observed value is smaller than the actual optical free mean path due to occlusion effects, which can be separated by an analytically derived equation [5]:

$$
\mu=\mu_{\mathrm{obs}}\left(1+\frac{1}{\cos \alpha}\right)
$$

(4.) Finally, the concentration at a given $z$-position can be related to the optical free mean path:

$$
c=\left(1-\frac{\mu}{W}\right) /\left(1+\frac{3}{2} \frac{\mu}{D}\right),
$$

where $W$ is a width of the flume and $D$ is a particle diameter [5].

\subsection{Sensitivity of outputs to method PARAMETERS}

There is a group of parameters associated with the employed instrumentation and its setting, such as minimum requirements on the camera and laser, exposure

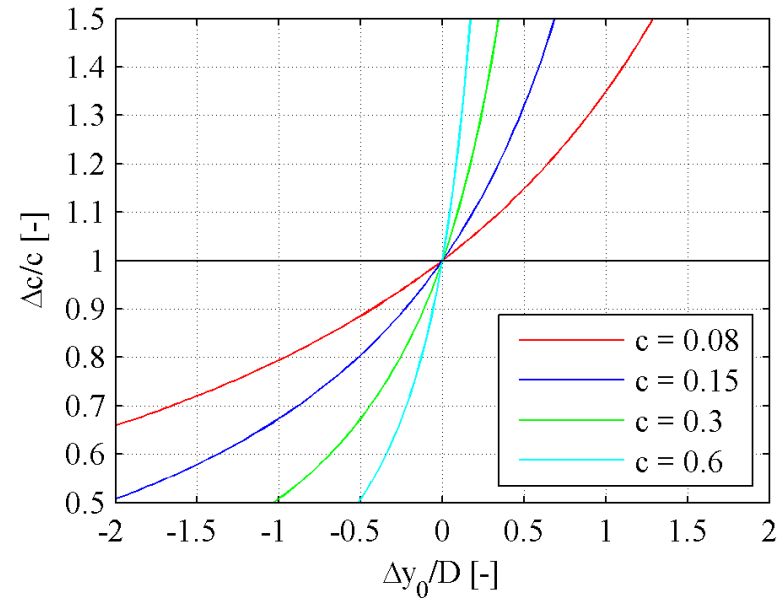

FiguRE 1. Theoretical relation between correction of wall position normalised by particle size and relative change of evaluated concentration.

time, frame rate, sufficient count of recorded images etc. These parameters were discussed in [5] and are out of the scope of the present study (except of the question of the image exposure touched below in $\S 4$ In the following paragraphs, we focus on parameters employed in the processing of the measured data.

Spinewine et al. [5] reported that even if holomorphic transformation is carefully calibrated, it is practically impossible to achieve an accuracy better than approximately one millimetre when projecting the mesh $[y, z]$ to the recorded images. As a result, position of the wall does not match the line $y=0$ and the evaluated $\mu_{\mathrm{obs}}$ is distorted. That's why Spinewine et al. 5] introduced a correction $\Delta y_{0}$ to wall position. The propagation of $\Delta y_{0}$ through (1) and (2) is shown in Figure 1 for the observation angle of $30^{\circ}$ and infinite flume width $W$. It can be seen that the error in the wall position became important particularly at high concentrations. This is because of the decreasing value of $\mu$ and hence the rising $\Delta y_{0} / \mu$. The decrease of $c$ with the increasing $\Delta y_{0}$ was observed above $\Delta y_{0} \approx 0$, when the full procedure of the data evaluation was applied to the measured data [6]. The change of the trend can be probably attributed to a distortion of the binning map caused by filtering out the hits localised at positions $y<\Delta y_{0}$ (only hits within the flume are counted to the binning map) the effect, which is not involved in the Figure 1 Because of the high sensitivity of the LSM method to the wall position, the correction $\Delta y_{0}$ has to be determined carefully. Spinewine et al. [5] evaluated $\Delta y_{0}$ from a shape of the binning map. We calculate the correction for each image employing a rectification procedure described later in $\S 3$. This enables us to correct potential changes of the measuring geometry of the camera and laser during the measurement.

The sensitivity of the evaluated concentration to an error in the observation angle follows directly from (1) and it is shown in Figure 2 for an observation angle of $\alpha=30^{\circ}$ and infinite flume width $W$. It is seen 


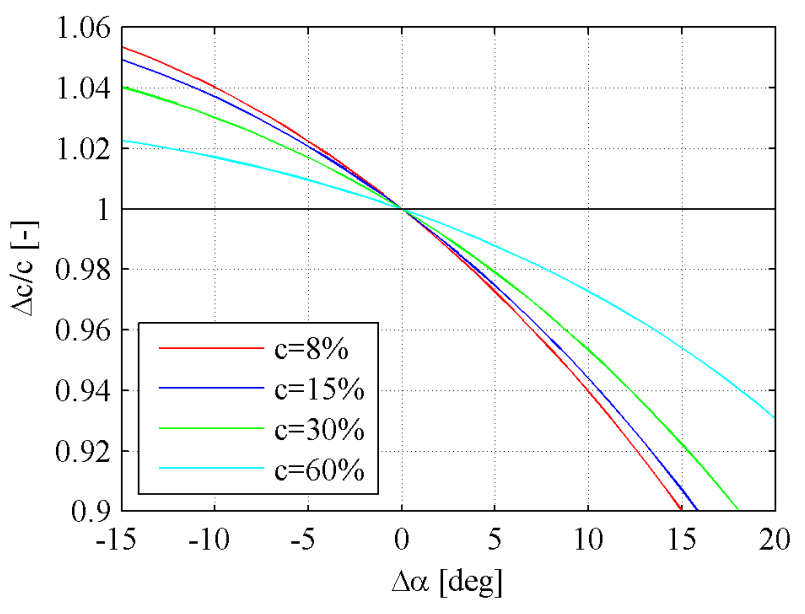

FiguRE 2. Theoretical relation between error in observation angle and relative change of evaluated concentration.

that the effect of $\Delta \alpha$ on the evaluated concentration is negligible when the angle is kept in the range of $25^{\circ}-35^{\circ}$ recommended in [5].

The effect of the threshold brightness for data filtering, $B_{\mathrm{th}}$, is difficult to analyse theoretically, because it is connected with the distribution of brightness in recorded images and this distribution follows from the way in which the laser light is refracted and reflected within the mixture of water and particles. A high sensitivity of concentration evaluated from the laser stripe measurement to the $B_{\mathrm{th}}$ was reported in [6]. Moreover, values of the optimal $B_{\text {th }}$ needed to obtain the correct concentration varied considerably with the concentration itself. No information on the determination of $B_{\text {th }}$ was supplied by the authors of the method in [5]. Effect of $B_{\mathrm{th}}$ will be demonstrated in $\$ 4$ using new experimental data. Here we only state that the absence of the guideline for the determination of $B_{\text {th }}$ can be considered as a serious weakness of the LSM method when the sensitivity of results to $B_{\text {th }}$ is high.

\section{EXPERIMENT IN FLUIDIZATION CELL}

\subsection{EXPERIMENTAL SETUP}

To investigate the effect of above discussed parameters experimentally, the LSM measurement was performed under controlled conditions within a fluidization cell. Height of the cell made of transparent Plexiglass was $105 \mathrm{~cm}$ and dimensions of the rectangular cross-section of the cell were $22 \times 20 \mathrm{~cm}$ (Figure 4 ). We used white plastics (POM) particles "Hostaform" to prepare a suspension of a known concentration within the cell. The particles were elliptical with mean dimensions of $3.8 \times 3.3 \times 2.6 \mathrm{~mm}$. Diameter of an equivalent sphere was $3.14 \mathrm{~mm}$ with a standard deviation of $0.1 \mathrm{~mm}$. The bed concentration of particles was 0.61 , density was $1357 \mathrm{~kg} / \mathrm{m}^{3}$ and settling velocity of individual particle was $0.13 \mathrm{~m} / \mathrm{s}$. Dilution of the suspension was controlled by setting a rate of an upward flow of water. A mean volumetric concentration of particles in the

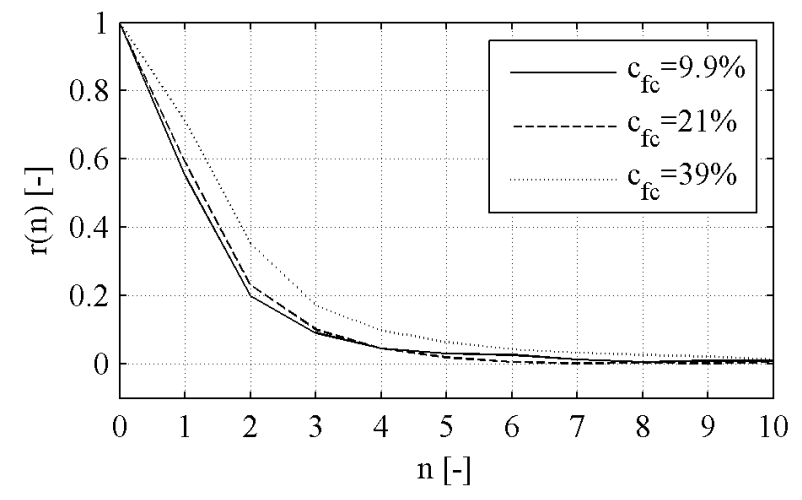

FigURE 3. Autocorrelation $r(n)$ of brightness evaluated at $y=\mu$.

suspension, $c_{\mathrm{fc}}$, was then calculated from a known weight of particles and measured volume occupied by the suspension. This volume - and hence the calculated concentration - fluctuated in time with a maximal deviation of $\pm 3 \%$ of the measured value. The LSM measurement was carried out at three distinct mean concentrations: $9.9 \%, 21 \%$ and $39 \%$.

A high speed camera (Dantec Dynamics FlowSense EO 4M-41 with an objective Carl Zeiss Planar 1.4/50 ZF) was used to observe the cell cross-section illuminated by the laser sheet. We used an image resolution of $1100 \times 2352 \mathrm{px}$, exposure time of $1.6 \mathrm{~ms}$, and frame rate of $28 \mathrm{~Hz}$. A typical image acquired by the camera is plotted in Figure 5 a. In order to avoid correlated data, the record was later resampled by including only each $n$th image in the analysis, where $n$ was set to 5 (see the autocorrelation function in Figure 3 ).

Seven distinct apertures $f / A$ were tested $(A=$ $5.7,6.7,8.0,9.5,11.3,13.5,16)$. The whole setup was covered by a curtain during measurements to ensure unchanged light conditions. The distance between the camera and the illuminated cross-section was approximately $50 \mathrm{~cm}$. Taking refraction at the air/plexiglass/water interfaces into account (see Figure 4 a), the inclination of camera view from the laser sheet plane was set to approximately $42^{\circ}$ to achieve an observation angle of $30^{\circ}$ (i.e. the value in the middle of the range of angles recommended in [5]). With the above described settings, one pixel of the recorded image corresponded to $0.15 \mathrm{~mm}$ on $y$ axis.

A diode source of a laser sheet of green colour (Blau Optoelektronik FP-Mvnano-520-30-30-F, $30 \mathrm{~mW}, 520 \mathrm{~nm}$ ) was mounted to the support enabling a precise adjusting of the laser position (Figure $4 \mathrm{~b}$ ). The distance between the laser and front wall of the cell was set to $34 \mathrm{~cm}$. A mirror fixed to the wall of the cell was used to reflect the laser sheet back to the laser when adjusting the orientation of the sheet perpendicular to the wall. The minimum thickness of the sheet achieved by focusing the laser was approximately $1 \mathrm{~mm}$, i.e. one third of the particle size (Figure 54). 

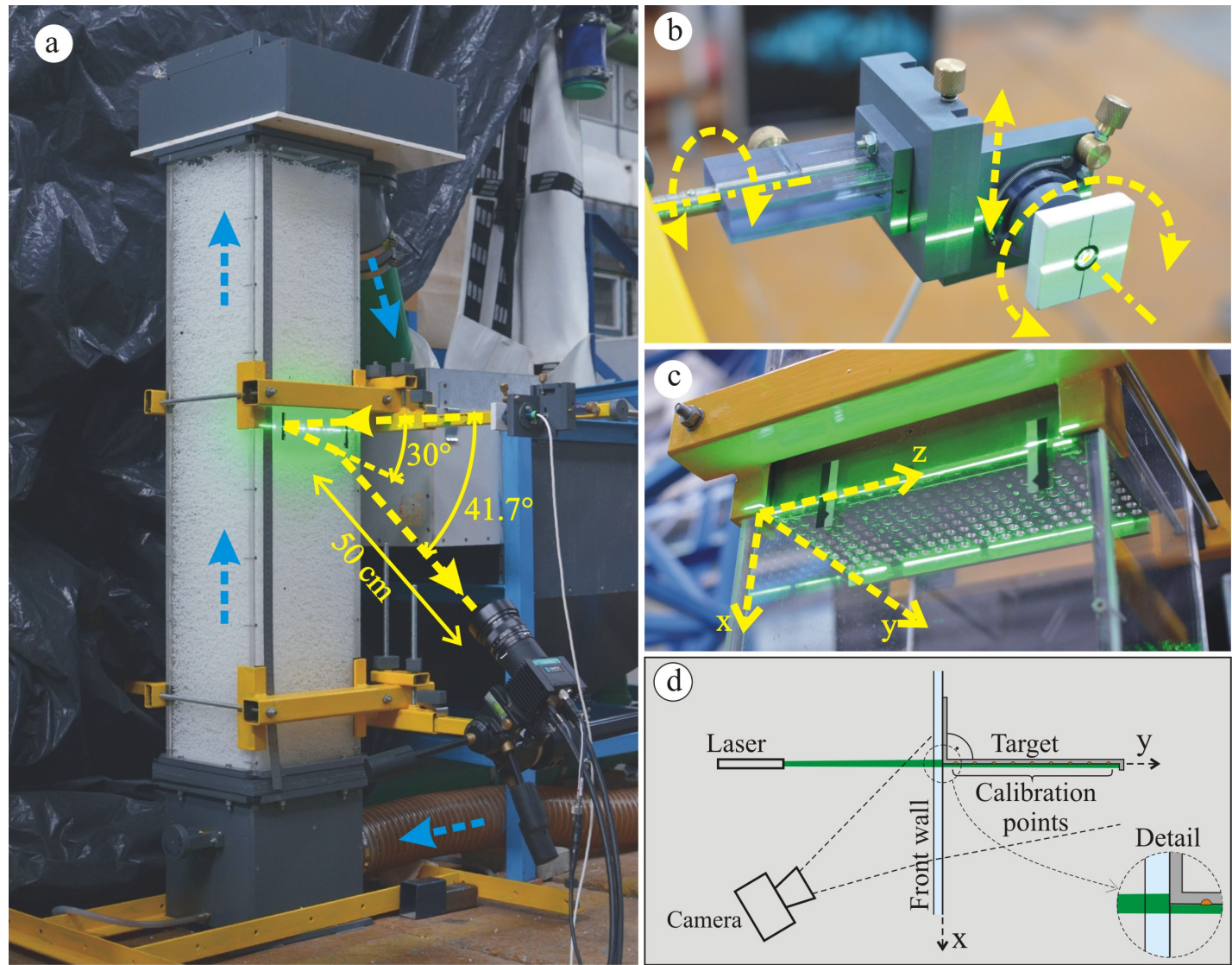

FiguRE 4. a) Fluidization cell with camera and laser support; b) detail of adjustable laser mounting with refracted laser sheet when setting laser orientations; c) calibration target and rectification stripes illuminated by laser; d) sketch of an experiment used for calibration of holomorphic transformation.
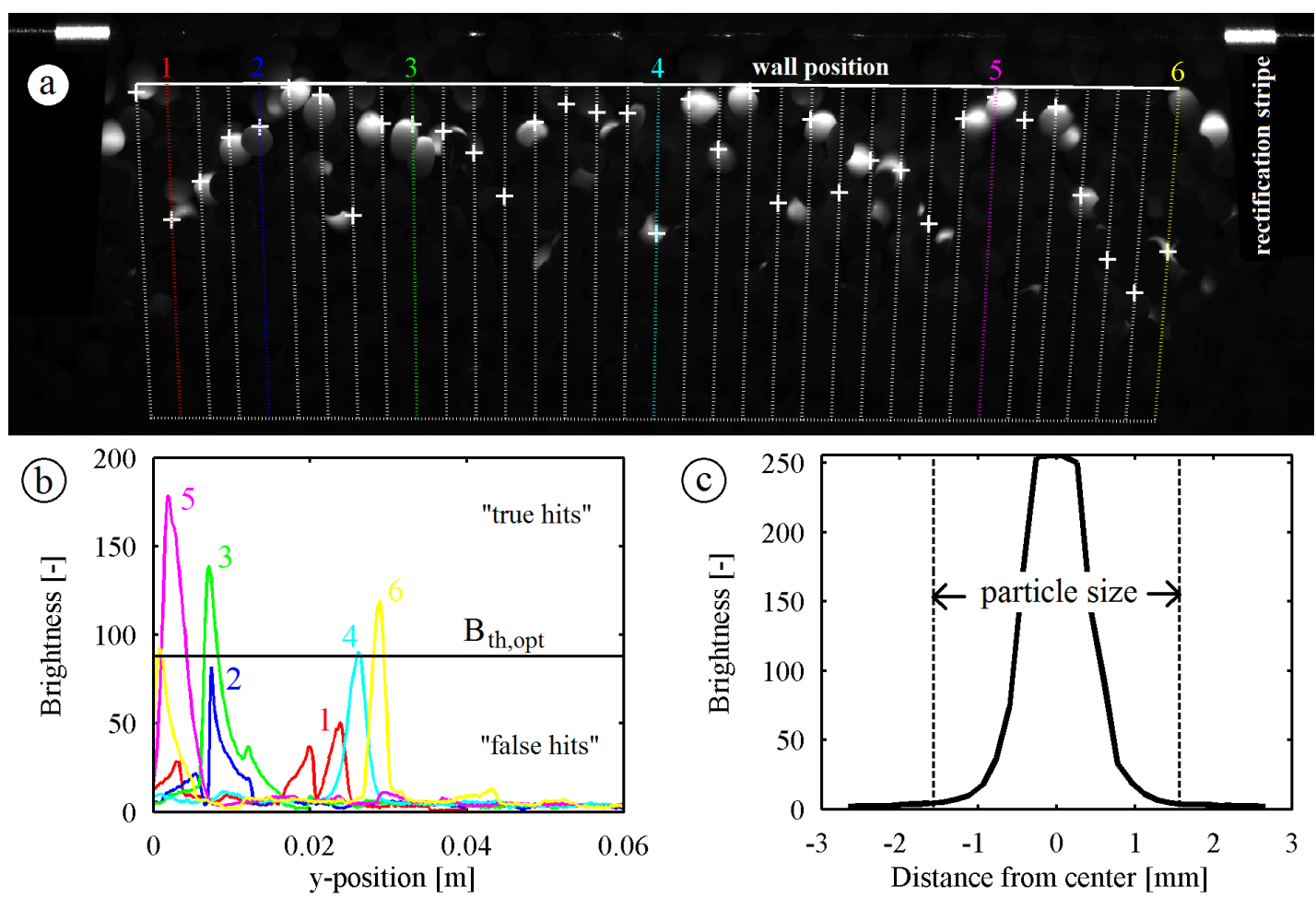

Figure 5. a) Typical image acquired by camera $(A=11.3, c=10 \%)$ with spots of maximum brightness identified along dotted lines; b) profiles of brightness at six z-positions highlighted in the panel a); c) distribution of brightness over an image of rectification stripe. 


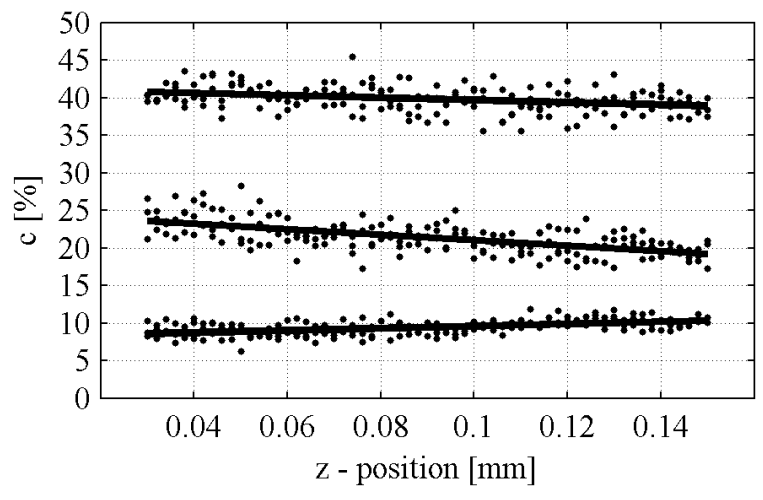

Figure 6. Concentrations evaluated at individual $z$-positions (points) fitted by a line.

The amount of data for statistics at each $z$-position is limited by the count of independent images recorded during the LSM. The concentration profile $c(z)$ evaluated from the LSM was considerably scattered because of insufficient data at each $z$-position. However, by fitting the profile by a line, it was possible to check the uniformity of the particle distribution across the cell. The observed variation of the concentration from one side of the cell to the other was within $\pm 1 \%$ in the cases of $c_{\mathrm{fc}}=9.9 \%$ and $c_{\mathrm{fc}}=39 \%$ and within $\pm 2 \%$ in the case of $c_{\mathrm{fc}}=21 \%$ (see Figure 6). To obtain a data set large enough for relevant statistics in a further analysis, we processed the data from all z-positions as a one data set assuming the profiles being uniform $(c(z)=$ const.). By doing this, we obtained $\sim 2 \cdot 10^{4}$ of identified $y$-positions of a point of a maximal brightness for each LSM measurement.

\subsection{Calibration}

An image of the calibration target was taken before filling the cell by particles to calibrate the holomorphic transformation. We used a target of the form of a plate perpendicular to the wall. When the target was attached to the inner surface of the wall, the position of the laser was carefully adjusted to illuminate the edge of the target (see Figure 4 c). Because the calibration points marked at our target laid in the illuminated cross-section, it was not necessary to extrapolate the calibration from the plane of the front wall as in the case of the target used in [5]. When a calibrated holomorphic transformation was used to project the line $y=0$ into the calibration image, the image of the line deviated from the observed edge of the calibration target maximally by $0.3 \mathrm{~mm}$.

To minimize the effect of error in the determination of the wall position discussed in $\S 2.2$, we fixed black and white labels to the wall of the cell. These labels with a light stripe drawn by the laser can be seen in Figure 4k. The position of a centre of the light stripes within the calibration image was calculated with a sub-pixel precision from

$$
\frac{\sum_{i} c_{i} \cdot B_{i}}{\sum_{i} B_{i}}
$$

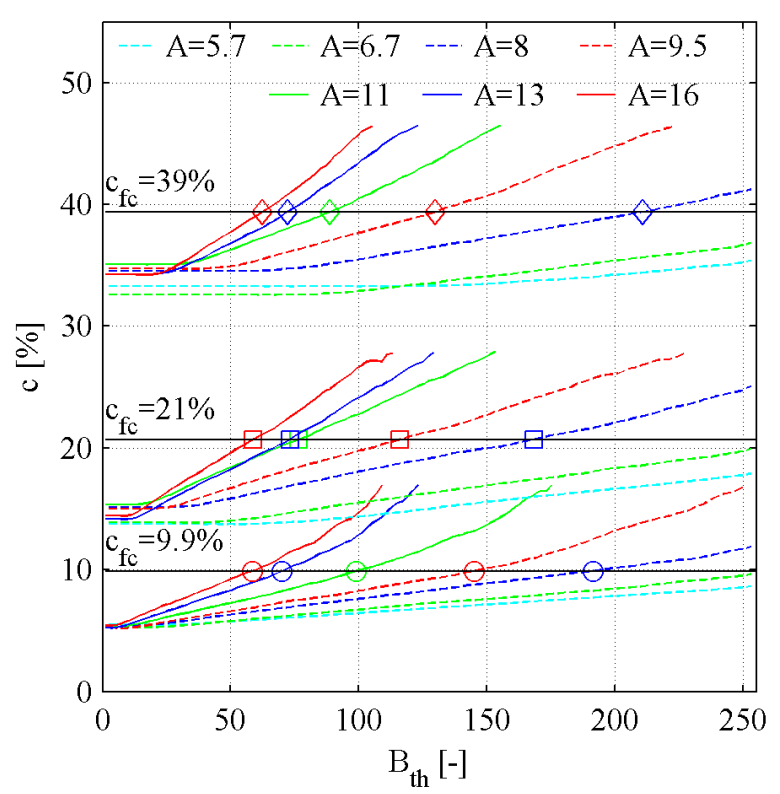

FiguRE 7. Relation between threshold brightness $B_{\text {th }}$ and concentration $c$ evaluated from LSM measurement. Colour lines - distinct apertures $f / A$ on camera; black horizontal lines - concentrations in fluidization cell; markers $-B_{\mathrm{th}, \mathrm{opt}}$ for given aperture and concentration.

where $B_{i}$ is the brightness of a pixel, $c_{i}$ is column index of the pixel, and $i$ is summation index of pixels occupying the image of the stripe. The same was also done for each image recorded during the LSM measurement. The discrepancy between positions of the stripes during the calibration and measurement was used to rectify the position of the recorded images with the sub-pixel accuracy.

\section{Results And Discussion}

Measurements at three distinct concentrations and seven apertures are evaluated and the results are plotted in a relation to the threshold $B_{\text {th }}$ in Figure 7 Using an 8-bit grey scale, $B_{\text {th }}$ varies between 0 and 255. Each curve in the figure corresponds to one experiment at a given concentration and aperture value and all laser hits satisfying $B>B_{\text {th }}$ are included in the statistics at a given $B_{\mathrm{th}}$. A strong relationship $c\left(B_{\mathrm{th}}\right)$ and wide range of optimal $B_{\text {th,opt }}$ is seen $\left(B_{\text {th,opt }}\right.$ is defined by $\left.c\left(B_{\mathrm{th}, \mathrm{opt}}\right)=c_{\mathrm{fc}}\right)$. Aperture obviously affects the scale of abscissa and hence the values of $B_{\text {th,opt }}$. From the increase of $c$ with increasing $B_{\mathrm{th}}$, it can be deduced that laser hits of low brightness are identified predominantly far away from the wall: increasing the value of $B_{\mathrm{th}}$ results in filtering out the distant hits, the mean position of remaining hits shifts closer to the wall, and the evaluated concentration increases. We also tested an improved method for an estimation of a mean free path from the measured data [2]. The improved method was expected to prevent the effect of the laser light attenuation by setting a maximum distance within which the hits are counted. Never- 


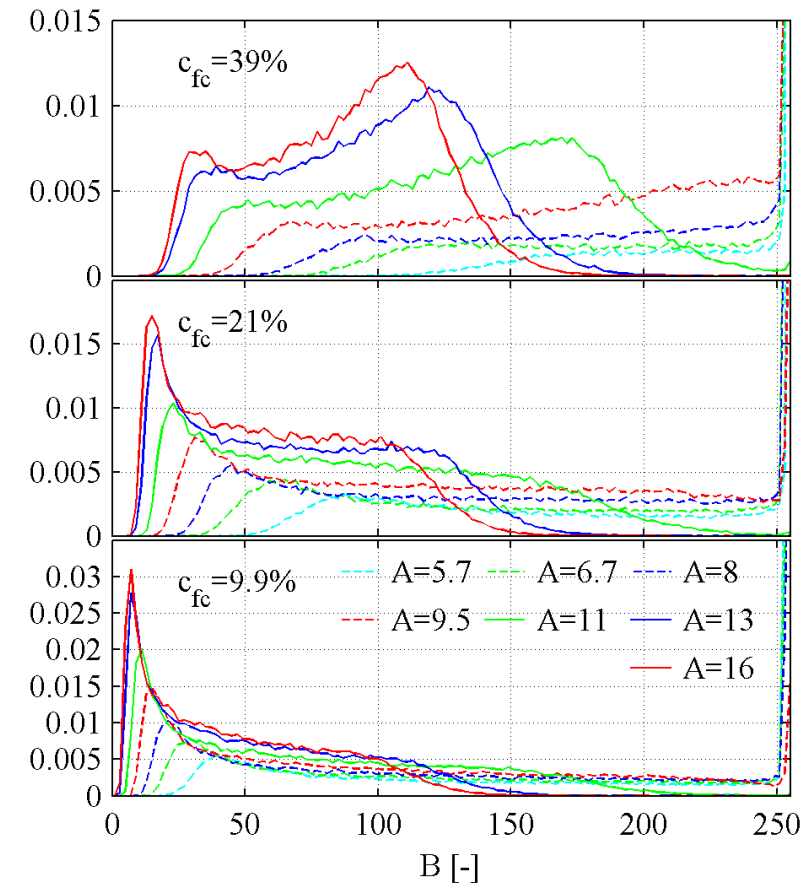

Figure 8. Histograms of hits brightness. Colour lines - distinct apertures on camera.

theless, we obtained qualitatively the same result although individual values of $B_{\text {th,opt }}$ shifted slightly, the sensitivity of the evaluated concentration to $B_{\text {th }}$ remained unchanged.

The role of $B_{\text {th }}$ in the data evaluation is to filter out bright points corresponding to the observation of the refracted and reflected laser sheet (i.e. "false" hits). Smooth course of curves in Figure 7 indicates that there is no sharp division between the "true" and "false" hits. This can also be deduced from observation of the wide range of maximum brightness values in Figure 5 b. However, the two groups of hits are expected to predominantly occupy distinct parts of brightness histogram forming two peaks. These histograms are plotted in Figure 8 (ordinates are normalized to give a unit area under the histograms). The histograms at the same concentration show a very similar course and differ only in vertical and horizontal scales. Expected peaks are recognized only at the highest concentration. However, let's try to identify position of these peaks. This can be done by fitting the histogram by two superposed probability density functions. As the $B_{\text {th }}$ must be positive, lognormal distribution is a natural choice. Parameters of the lognormal distribution can be conveniently fitted when a transformation to normal coordinates is performed. After the transformation, the two peaks can be clearly recognized at all concentrations (Figure 9). Further analysis showed that the lognormal distribution (i.e. normal distribution in the normal coordinates) is not suitable to fit the histograms - a very good fit was achieved when the two triangles were used in the normal coordinates.

The histograms for the measurements with an aper-

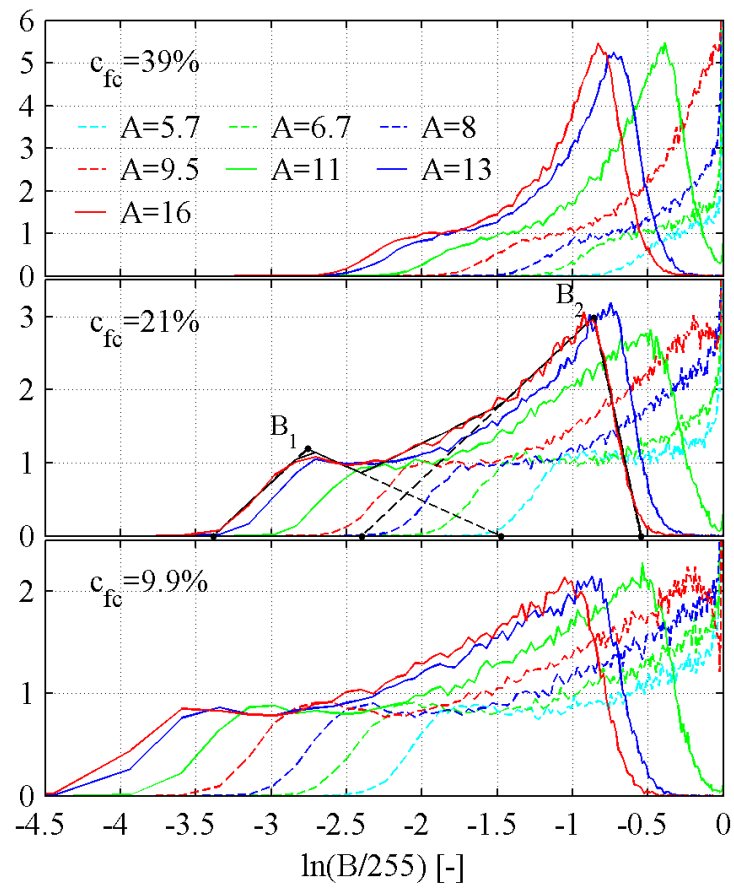

FiguRE 9. Histograms of hits' brightness transformed to normal coordinates. Colour lines - distinct apertures on camera; black line - triangles fitting the histogram at $c_{\mathrm{fc}}=21 \%$ and $A=16$.

ture higher than $f / 9.5$ cannot be fitted because the second peak lies behind the range of $B$ - corresponding parts of the histograms are shrunk to the value of $B=255$ in Figures 8 . These measurements are excluded from the further analysis. As $B_{\text {th }}$ should be used to distinguish between "true" and "false" hits, it should be related to the position of the peaks. This hypothesis is proved in Figure 10, where histograms are plotted in coordinates normalised by $B_{2}$ - the position of the second peak. It can be seen that: 1) all the histograms at the same concentration fall to the same curve after the transformation; 2) the curve is successfully fitted by the two triangles (after the transformation back from the normal coordinates, triangles are distorted to black curves); 3) when the values of $B_{\text {th,opt }}$ deduced from Figure 7 are normalised by $B_{2}$, they fall close to the mean value of $0.57 ; 4$ ) normalised $B_{\text {th,opt }}$ falls to the region where the two peaks overlap.

Based on this observation, the following guideline for the evaluation of the threshold $B_{\text {th }}$ can be proposed: the brightness of laser hits has to be within the range of the grey scale recorded by the camera (aperture, exposure time and ISO have to be set carefully); then, the histograms of hits' brightness can be fitted by triangles in normalized coordinates; $B_{\mathrm{th}}$, is obtained by multiplying the position of the peak of the second triangle, $B_{2}$, by the factor 0.57 . The predicted values of $B_{\text {th }}$ are compared with $B_{\text {th,opt }}$ in Figure 11, showing a reasonable match. If the measured data are processed with the predicted $B_{\mathrm{th}}$, the evaluated concentrations match those measured in 


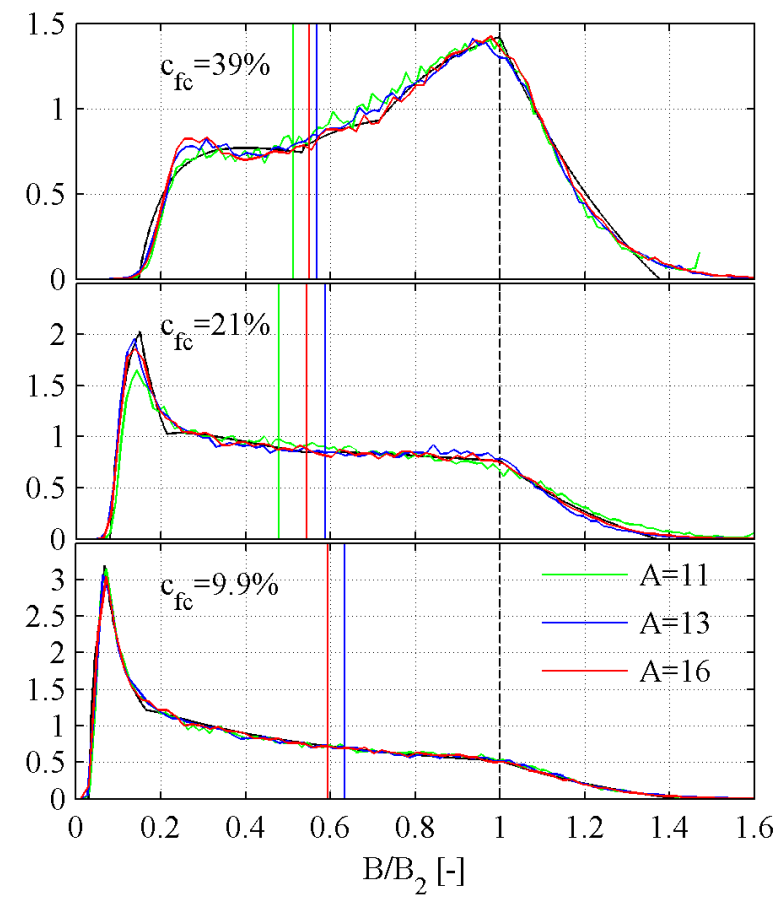

FiguRE 10. Histograms of hits' brightness normalised by position of the second peak. Colour lines - distinct apertures on camera; black line - fit of the two peaks (triangles in normal coordinates); vertical lines - normalised values of $B_{\mathrm{th} \text {,opt }}$.

the fluidization cell perfectly (Figure 12). The good match indicates that both crucial parameters of the method, $B_{\text {th }}$ and $\Delta y_{0}$, were treated correctly.

The particles we tested were almost spherical - the shape for which the LSM method was derived. However, authors of the method showed that it works also with non-spherical particles 5 . Shape of a particle, as well as optical properties of a particle material in respect to a laser light (reflectivity and opacity), is expected to affect mutual positions and shapes of peaks recognized in the brightness histogram. Hence, the optimal value of $B_{\mathrm{th}} / B_{2}$ ratio has to be determined from calibration tests for each combination of particles and the laser employed. Our results show that then it is virtually independent of the concentration and within certain range - exposure.

It should be noted that the typical image of particles hit by a laser obtained by [5] differs from our image in Figure $5 \mathrm{a}$ - the laser stripe was narrower and sharper in [5]. This can be explained by different optical properties of tested particles. Our particles are probably partially translucent whereas those used in [5] are not. When perfectly opaque particles are used, the false hits can diminish and the laser stripe measurement becomes insensitive to $B_{\mathrm{th}}$, in a wide range of brightness values.

\section{Conclusions}

Three parameters enter the process of an evaluation of the laser stripe measurement (LSM). While the

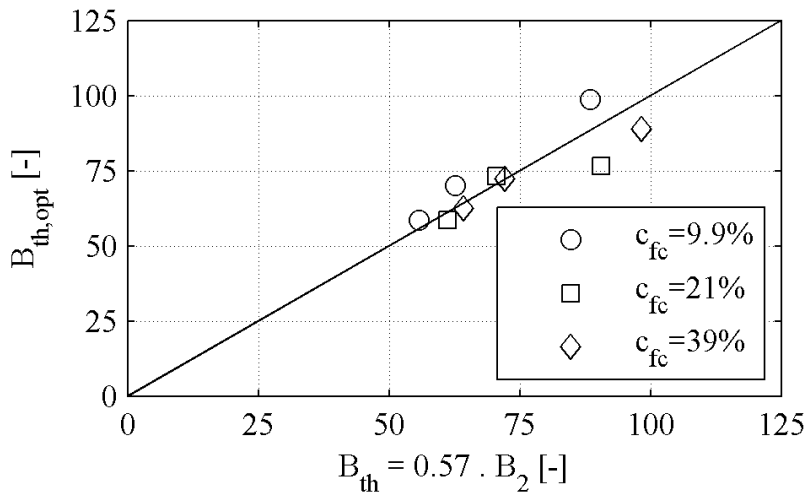

Figure 11. Comparison of predicted threshold brightness with the optimal values needed to obtain correct concentration.

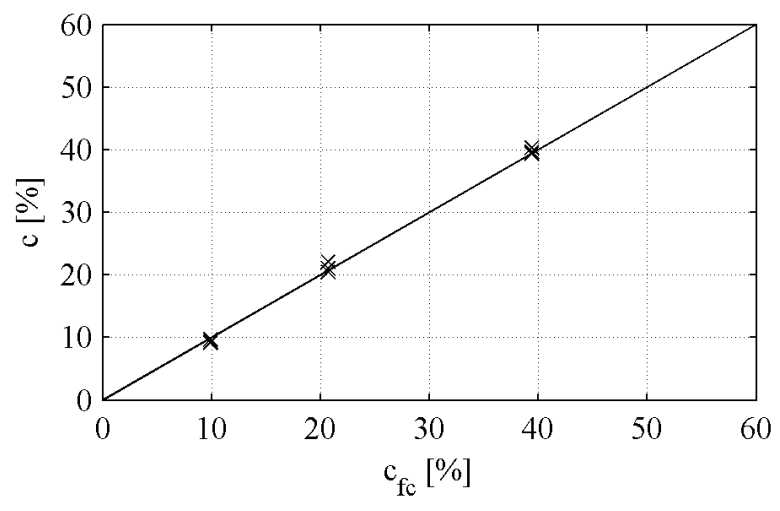

Figure 12. Comparison of concentration evaluated from LSM measurement with concentration measured in fluidization cell (only measurements with $A>9.5$ included).

observation angle affects the measurement results insignificantly, the threshold brightness for the data filtering and the wall position correction are found to play much more important roles. We calculated the wall position correction from the position of the light stripes drawn by the laser sheet on the rectification labels stuck to the wall of a conduit. This procedure seems to provide sufficiently accurate results. The LSM is expected to be practically insensitive to the threshold brightness if particles and laser optical properties are ideal (particularly the particle opacity). However, for partially translucent particles, it appears to be very threshold-brightness sensitive. A need for guidelines for a determination of the threshold arises in the latter case. We attempted to relate the threshold to the shape of a histogram of the brightness of the laser hits evaluated from our measurements in a fluidization cell. We found that two peaks can be distinguished in the histogram. The first peak can be attributed to "false hits", which have to be filtered out and the second one to the "true" observations of particles hit by the laser sheet. A perfect match is achieved between concentrations evaluated from the LSM and actual concentrations within the flu- 
idization cell provided that the threshold is related to the second peak position scaled by a factor of 0.57 . Assumingly, particular values of the factor may be sensitive to parameters associated with the properties of the particle and laser.

\section{ACKNOWLEDGEMENTS}

The research has been supported by the Czech Science Foundation through the grant project No. 16-21421S.

\section{REFERENCES}

[1] Berzi, D.: Analytical Solution of Collisional Sheet Flows. Journal of Hydraulic Engineering-ASCE, Vol. 137, No. 10, pp. 1200-1207, 2011. ISSN: 0733-9429. DOI:10.1061/(ASCE)HY.1943-7900.0000420

[2] Capart, H., Fraccarollo, L.: Transport Layer Structure in Intense Bed-Load. Geophysical Research Letters, Vol. 38, Art. No. L20402, 2011. ISSN: 0094-8276. DOI:10.1029/2011GL049408
[3] Berzi, D., Fraccarollo, L.: Inclined, Collisional Sediment Transport. Physics of Fluids, Vol. 25, No. 10, Art. No. 106601, 2013. ISSN: 1070-6631. DOI:10.1063/1.4823857

[4] Matoušek, V., Zrostlík, Š.: Laboratory Testing of Granular Kinetic Theory for Intense Bed Load Transport. Journal of Hydrology and Hydromechanics, Vol. 66, No. 3. (in press). DOI:10.2478/johh-2018-0012

[5] Spinewine et al.: Laser Stripe Measurements of Near-Wall Solid Fraction in Channel Flows of Liquid-Granular Mixtures. Experiments in Fluids, Vol. 50, No. 6, pp. 1507-1525, 2011. ISSN: 0723-4864. DOI:10.1007/s00348-010-1009-7

[6] Krupička et al.: Validation of Laser-Penetration- and Electrical-Conductivity-Based Methods of Concentration Measurement in Flow with Intense Transport of Coarse Sediment. Proceedings of the International Conference Experimental Fluid Mechanics 2017, Mikulov (Czech Republic), 2017, pp. 322-327 